\section{Visual selection of features and objects: Is location special? A reinterpretation of Nissen's (1985) findings}

\author{
CLAUS BUNDESEN \\ Copenhagen University, Copenhagen, Denmark
}

Nissen (1985) compared selection by location with selection by color or shape in partial-report experiments. Her analysis of response contingencies when a target was defined in terms of one attribute (location, color, or shape), and when the task was to report the two remaining attributes, suggested a special role for selection by location: It appeared that cross-referencing between color and shape was mediated by location. An alternative interpretation is developed here: The findings are explained by a theory of attention (Bundesen, 1990), in which selection by location is treated on a par with selection by color or shape.

In Attention and Performance XI, Nissen (1985) reported an intriguing comparison between selection by location and selection by color or shape. Nissen's data showed that selecting an element by location and reporting its color was no more efficient than selecting the element by color and reporting its location. However, her analysis of response contingencies when a target was defined by one attribute (location, color, or shape), and when the task was to report the two remaining attributes, suggested a special role for selection by location: It appeared that cross-referencing between color and shape was mediated by location.

Nissen's (1985) results have been regarded as strong evidence for the view that selection by location is governed by principles different from those that govern selection by color or shape (see, e.g., LaBerge \& Brown, 1989, p. 106). I shall present a reinterpretation of Nissen's findings. The reinterpretation will show how the findings can be explained by a theory of attention (Bundesen, 1990), in which selection by location is treated on a par with selection by color or shape. (See Duncan, 1980, for a related interpretation of the results of von Wright, 1968, 1970.)

\section{Nissen's (1985) Findings}

Nissen (1985) compared selection by location with selection by color and shape in two partial-report experiments. In both experiments, every stimulus display consisted of four colored shapes, each subtending about $0.3^{\circ}$, which were centered about $0.8^{\circ}$ above, below, to the left, and to the right of the center of the display. The shapes were a circle, a square, a triangle, and a diamond; the colors were red, green, blue, and black. No color or shape was repeated within any display. The exposure duration was

Correspondence concerning this article should be addressed to Claus Bundesen, Psychological Laboratory, Copenhagen University, Njalsgade 90, DK-2300 Copenhagen S., Denmark. about 130 msec. The offset of the display was followed immediately by a pattern mask and a selection cue.

In Experiment 1, the cue was the name of a location or the name of a color. When the cue was the name of a location, the task was to report the color that had appeared at that location, and when the cue was the name of a color, the task was to report the location at which the color had appeared. The results showed that the probability of reporting the location of an element correctly, when the color was given as a cue, was approximately the same as the probability of reporting the color correctly when the location was given as a cue. In Nissen's symbols,

$$
P(L \mid C)=P(C \mid L) \text {. }
$$

Experiment 2 had two main conditions. In Condition 1 , a location cue was used, and the task was to report both the color and the shape of the element that had appeared at the cued location. The probability of reporting both features of the cued element correctly was approximately the same as the product of the probability of reporting the color correctly and the probability of reporting the shape correctly (for a replication and extension, see Isenberg, Nissen, \& Marchak, 1990). In symbols,

$$
P(C \& S \mid L)=P(C \mid L) P(S \mid L) \text {. }
$$

In Condition 2, a color cue was used, and the task was to report both the location and the shape of the element in the cued color. ${ }^{1}$ In this case, the probability of reporting both features of the cued element correctly differed from the product of the probability of reporting the location correctly and the probability of reporting the shape correctly. Nissen hypothesized that, when presented with a color cue, the subject first determined the location of the element in the cued color with probability $P(L \mid C)$ and then determined the shape of the element at that location with probability $P(S \mid L)$. According to this hypothesis, the probability of reporting both features of the cued element correctly, $P(L \& S \mid C)$, should be given by

$$
P(L \& S \mid C)=P(L \mid C) P(S \mid L),
$$

and the results supported this prediction.

Nissen further hypothesized that, on trials on which the location response was incorrect, the subject attempted to report the shape at the (incorrectly) selected location. That attempt succeeded with probability $P(S \mid L)$. On the remaining incorrect location trials, shape responses were assumed to be uniformly distributed among the remaining three alternatives, one of which was the correct shape response. Accordingly, the probability of correct shape given incorrect location was predicted to be $[1-P(S \mid L)] / 3$, and the predicted joint probability of incorrect location and correct shape, $P(\bar{L} \& S \mid C)$, was given by

$$
P(\bar{L} \& S \mid C)=[1-P(L \mid C)][1-P(S \mid L)] / 3 .
$$

Again the results supported the prediction. 


\section{Theoretical Reinterpretation}

In the theory described by Bundesen (1990), visual recognition and attentional selection consist in making perceptual categorizations of the form " $x$ belongs to $F$," where $x$ is an element in the visual field and $F$ is a perceptual category. An element in the visual field is a perceptual unit in the sense given, for instance, in Kahneman (1973, p. 68) and in Woodworth (1938, p. 625). Examples of perceptual categories are the class of red elements, the class of circular elements, and the class of elements located above fuxation.

Assume that, during stimulus exposure, perceptual categorizations with respect to color, shape, and location were sampled independently (cf. Bundesen, 1990, p. 528). Assume that, in every experimental condition, the attentional weights (see Bundesen, 1990; Rumelhart, 1970) of the four elements in the stimulus display were equally great, so that attentional weighting can be neglected. Assume that short-term storage capacity permitted at least four elements (see Bundesen, 1990; Sperling, 1960), so that storage limitations can be neglected. Let the probability of sampling the correct color categorization of an element be a constant $p_{C}$, let the probability of sampling the correct shape categorization of an element be a constant $p_{S}$, and let the probability of sampling the correct location categorization of an element be a constant $p_{L}$. Suppose perceptual confusion errors (sampling of incorrect categorizations) were negligible. Finally, suppose location was special in only the following respect: To a good approximation, $p_{L}$ was 1 (i.e., the location categorizations required by the task were extremely fast).

Consider Experiment 1. Let $x_{i}(i=1,2,3$, or 4$)$ be one of the four elements in a stimulus display, and let $C_{i}$ be the color and $L_{i}$ the location of element $x_{i}$. When cued with location $L_{i}$, the subject determined $x_{i}$ with probability 1 (the probability that the categorization " $x_{i}$ belongs to $L_{i}$ " had been sampled). If the categorization " $x_{i}$ belongs to $C_{i}$ " had been sampled, then the subject reported $C_{i}$ with probability 1 . Otherwise, the subject made a guess and reported $C_{i}$ with probability $1 / 4 .{ }^{2}$ Hence,

$$
P\left(C_{i} \mid L_{i}\right)=p_{C}+\left(1-p_{C}\right) / 4 .
$$

Conversely, when cued with color $C_{i}$, the subject determined $x_{i}$ without guessing provided the categorization " $x_{i}$ belongs to $C_{i}$ " had been sampled. Otherwise, the subject guessed $x_{i}$ with probability $1 / 4$. Given $x_{i}$, the subject reported $L_{i}$ with probability 1 . Thus,

$$
\begin{aligned}
P\left(L_{i} \mid C_{i}\right) & =p_{C}+\left(1-p_{C}\right) / 4 \\
& =P\left(C_{i} \mid L_{i}\right),
\end{aligned}
$$

which explains Equation 1. Note that, by a similar argument,

$$
\begin{aligned}
P\left(L_{i} \mid S_{i}\right) & =p_{S}+\left(1-p_{S}\right) / 4 \\
& =P\left(S_{i} \mid L_{i}\right),
\end{aligned}
$$

where $S_{i}$ is the shape of element $x_{i}$.
Consider Condition 1 of Experiment 2. When cued with location $L_{i}$, the subject determined $x_{i}$ with probability 1 . Given $x_{i}, C_{i}$ was correctly reported with probability $p_{C}+\left(1-p_{C}\right) / 4$. Independently of whether $C_{i}$ was correctly reported, $S_{i}$ was correctly reported with probability $p_{s}+\left(1-p_{s}\right) / 4$. Hence,

$$
\begin{aligned}
P\left(C_{i} \& S_{i} \mid L_{i}\right) & =\left[p_{C}+\left(1-p_{C}\right) / 4\right]\left[p_{S}+\left(1-p_{S}\right) / 4\right] \\
& =P\left(C_{i} \mid L_{i}\right) P\left(S_{i} \mid L_{i}\right)
\end{aligned}
$$

(cf. Equations 5 and 6), which explains Equation 2.

Consider Condition 2 of Experiment 2. When cued with color $C_{i}$, the subject determined $x_{i}$ with probability $p_{C}+\left(1-p_{C}\right) / 4$. Given $x_{i}$, the subject reported $L_{i}$ with probability 1 and $S_{i}$ with probability $p_{s}+\left(1-p_{s}\right) / 4$. Accordingly,

$$
\begin{aligned}
P\left(L_{i} \& S_{i} \mid C_{i}\right) & =\left[p_{C}+\left(1-p_{C}\right) / 4\right]\left[p_{S}+\left(1-p_{S}\right) / 4\right] \\
& =P\left(L_{i} \mid C_{i}\right) P\left(S_{i} \mid L_{i}\right),
\end{aligned}
$$

which explains Equation 3.

With probability $\left(1-p_{C}\right) 3 / 4$, the categorization " $x_{i}$ belongs to $C_{i}$ " had not been sampled and the subject guessed an element different from $x_{i}$. In such cases, location responses were incorrect, but with probability $\left(1-p_{S}\right) / 4$, the subject reported $S_{i}$ by guessing. Thus, the predicted joint probability of incorrect location and correct shape is

$$
P\left(\overline{L_{i}} \& S_{i} \mid C_{i}\right)=\left[\left(1-p_{C}\right) 3 / 4\right]\left(1-p_{S}\right) / 4 \text {. }
$$

By Equations 5 and 6, Equation 7 reduces to

$$
P\left(\overline{L_{i}} \& S_{i} \mid C_{i}\right)=\left[1-P\left(L_{i} \mid C_{i}\right)\right]\left[1-P\left(S_{i} \mid L_{i}\right)\right] / 3,
$$

which explains Equation 4.

\section{Conclusion}

Nissen's (1985) findings are consistent with the view that selection by location is governed by the same principles as selection by color or shape. In particular, the findings can be explained by assuming mutual independence between perceptual categorizations with respect to color, shape, and location (cf. Bundesen, 1990). In the suggested explanation, location was special in only the following sense: The location categorizations required by the experimental task were extremely fast.

\section{REFERENCES}

Bundesen, C. (1990). A theory of visual attention. Psychological Review, 97, 523-547.

DUNCAN, J. (1980). The locus of interference in the perception of simultaneous stimuli. Psychological Review, 87, 272-300.

IsenBerg, L., NisSen, M. J., MARChak, L. C. (1990). Attentional processing and the independence of color and orientation. Journal of Experimental Psychology: Human Perception \& Performance, 16, 869-878.

Kahneman, D. (1973). Attention and effort. Englewood Cliffs, NJ: Prentice-Hall.

LABERge, D., \& Brown, V. (1989). Theory of attentional operations in shape identification. Psychological Review, 96, 101-124.

Nissen, M. I. (1985). Accessing features and objects: Is location special? In M. I. Posner \& O. S. M. Marin (Eds.), Attention and performance $X I$ (pp. 205-219). Hillsdale, NJ: Erlbaum. 
RumelharT, D. E. (1970). A multicomponent theory of the perception of briefly exposed visual displays. Journal of Mathematical Psy chology, 7, 191-218.

SPERLING, G. (1960). The information available in brief visual presentations. Psychological Monographs, 74 (11, Whole No. 498).

VON WRIGHT, J. M. (1968). Selection in visual immediate memory Quarterly Journal of Experimental Psychology, 20, 62-68.

voN WRIGHT, J. M. (1970). On selection in visual immediate memory Acta Psychologica, 33, 280-292.

Woodworth, R. S. (1938). Experimental psychology. New York: Holt.

\section{NOTES}

1. Conditions 1 and 2 were run with the same subjects on separate days. The subjects also performed a shape-cue condition on a separate day. The results from the shape-cue condition "were consistent with the results from the color-cue condition" (Nissen, 1985, n. 1).

2. Assuming random guessing among the four possibilities simplifies the exposition. Alternatively, one could assume sophisticated guessing, that is, random guessing among only those possibilities that were consistent with all of the sampled categorizations (including categorizations of elements other than $x_{i}$ ). Assuming sophisticated guessing would complicate the exposition but not affect the conclusions.

(Manuscript received November 26, 1990; revision accepted for publication February 5, 1991.) 